\section{The Principle of Activity and Lagrange's Equations. Rotation of a Rigid Body.}

THERE are some people who understand by Newton's second law of motion nothing more than the three equations of motion for a body which can be treated as a particle, viz., $m \ddot{x}=\mathrm{X}$, sc. (or rather the equivalent equations for impulsive forces). Such people, however, would probably not seriously object to any dynamical truth whatsoever, from the conservation of energy to the principle of varying action, being read into this law, if only he who does so would explain clearly his own in terpretation of Newton's statement. I, for one, am a little curious to have stated fully the principle which justifies Mr. Heaviside in his letter in your issue of January 29 in deducing from the solitary equation

$$
\dot{\mathrm{T}}=\left(\frac{d}{d t} \cdot \frac{d \mathrm{~T}}{d z_{1}}-\frac{d \mathrm{~T}}{d x_{1}}\right) v_{1}+\ldots
$$

that " by Newton, the force on $x_{1}$ is the coefficient of $v_{1}$."

It is a sufficient indication either of an incorrect premiss or of bad logic, however obscure an argument may be, if the conclusion be wrong ; one does not readily see from Mr. Heaviside's letter how he could object to his method being applied directly to the motion of a rigid body with one point fixed, in which case, as is well known, taking

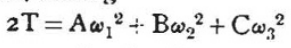

it leads to a wrong expression for the external couple round the

axis of $x$, viz. $A \dot{\omega}_{1}$ instead of the correct one, A $\dot{\omega}_{1}-(B-C) \omega_{2} \omega_{3}$.
W. MCF. ORR.

Royal College of Science, Dublin, February 2.

PROF. ORR's opening remarks perhaps indicate that the want of appreciation of Newton's dynamics is even greater than I supposed. My authority for Newton is that stiff but thoroughgoing work, Thomson and Tait. On comparison, I find that Prof. Orr's "some people" seem to overlook the vitally im. portant third law, without which there could be no dynamics resembling the reality, and also the remarkable associated scholium "Si restimatur...," enunciating the principle of activity, which is of such universal and convenient application, both by practicians and by some theorists. In my short outline of the beginning of the theory of I agrange's equations, my argument " by Newton" referred to the activity principle.

The example of failure given by Prof. Orr is remarkable in more than one way. If the three coordinates specified the configuration, then the equations of motion would come out in the way indicated. It is clear, therefore, from the failure that in the concrete example of a rotating rigid body, the coordinates employed, which are the time-integrals of the angular velocities about three moving axes, are not proper Lagrangian coordinates within the meaning of the Act. If we use coordinates which do fix the configuration (Thomson and Tait, \$319), there is no failure.

But it is quite easy to avoid the usual complicated trigonometrical work, and obtain the proper equations of motion by allowing for the motion of the axes. Thus, if $\mathrm{a}$ is the angular velocity, the angular momentum is

$$
\mathbf{i} \frac{d \mathrm{~T}}{d a_{1}}+\ldots=\mathrm{A} a_{1} \mathbf{i}+\mathrm{B} a_{2} \mathrm{j}+\mathrm{C} a_{3} \mathbf{k},
$$

and the torque is its time differentiant, that is,

$$
\mathbf{F}=\mathrm{A} \dot{a}_{1} \mathbf{i}+\mathrm{B} \dot{a}_{2} \mathbf{j}+\mathrm{C} \dot{a}_{3} \mathbf{k}+\mathrm{A} a_{1} \frac{d \mathbf{i}}{d t}+\mathrm{B} a_{2} \frac{d \mathbf{j}}{d t}+\mathrm{C} \alpha_{3} \frac{d \mathbf{k}}{d t} .
$$

- Here $\mathbf{i}, \mathbf{j}, \mathbf{k}$ are unit vectors specifying the directions of the principal axes. They only vary by the rotation, so $d \mathbf{i} / d t=\mathrm{Vai}$, $\& c$, and this makes

$\mathbf{F}=\mathrm{A} a_{1}\left(\mathbf{j} a_{3}-\mathbf{k} a_{22}\right)+\mathrm{B} a_{32}\left(\mathbf{k} a_{1}-\mathbf{i} a_{3}\right)+\mathrm{C} a_{3}\left(\mathbf{i} a_{2}-\mathbf{j} a_{3}\right)+\mathrm{A} \dot{a}_{1} \mathbf{i}+\ldots$ $=\mathbf{f}\left\{\mathrm{A} \dot{a}_{1}-a_{2} a_{3}(\mathrm{~B}-\mathrm{C})\right\}+\mathbf{j}\{\ldots \hat{\mathbf{k}}\{\ldots\}$.

This exhibits Euler's three well-known equations of motion round the three principal moving axes.

In general, $\mathrm{T}=\frac{1}{2} \mathrm{a} . \mathbf{M a}$, where $\mathrm{M}$ is a vectorial matrix (or linear vector operator), fixed in the body. Then the momentum is $\mathrm{Ma}$, and the torque is

$$
\mathbf{F}=M \dot{\mathbf{a}}+\dot{M} \mathbf{a}=M \dot{a}+(V \mathbf{a} M) \mathbf{a} .
$$

This allows MI to be specified with respect to any axes fixed in the rotating boily. Of course, the principal axes are the best. I may refer to my " Elec. Pa.," vol. ii., p. 547, footnote, for No. 1738 , voI. 67$]$ details of a similar calculation relating to the torque (and activity thereof) produced in an eolotropic dielectric under electric stress.

The following concisely exhibits the necessity of allowing for variation of $\mathrm{M}$, and how it is done in the general case of $n$ independent variables:-Let $T=\frac{1}{2} \nabla \cdot M \nabla=\frac{1}{2} p v$. Then $\mathbf{v}$ is a "vector" or complex of $n$ velocities, and $\mathbf{p}=\mathrm{Mv}$ is the corresponding momentum, whilst $\mathrm{M}$ is a symmetrical matrix. By differentiation to $t$,

$$
\begin{aligned}
& \dot{\mathrm{T}}=\mathbf{v}\left(\mathbf{M} \dot{\mathbf{v}}+\frac{1}{2} \dot{M} \mathbf{v}\right)=\mathbf{F} \mathbf{v} \quad \text { (Hamilton) } \\
& \mathrm{T}=\mathbf{v}\left(\dot{\mathbf{p}}-\frac{1}{2} \dot{\mathbf{M}} \mathbf{v}\right)=\mathbf{F} \mathbf{v} \quad \text { (Lagrange). }
\end{aligned}
$$

Here $\mathbf{F}$ is the force on the system, in the same sense as $\mathbf{\nabla}$ is the velocity of the system. For $\dot{M}$ substitute $\left.v_{(}^{\prime} d \mathrm{M} / d x\right)$, to come to the usual forms by breaking up into $n$ components. But the above are more general, because $M$ may vary inde pendently of $x$. Activity should be the leading idea.

Oliver IHEAVISIDE.

\section{Insects and Petal-less Flowers.}

I was much interested by Mr. Bulman's account of Prof. Plateau's experiments in the matter of insects' visits to petalless flowers in the issue of NATERE for February 5 (p. 319 ), wherein it is stated that Prof. Plateau contends that insects " are not attracted by the brilliant colours of the blossoms, but rather by the perception in some other way-probably by scent-- that there is honey or pollen."

It has not been my good fortune to read Prof. Plateau's own account of the experiments which led him to the above conclusion, but it certainly appears to me, from your correspondent's summary, that he is generalising from an observation which has only a strictly limited application.

We are told that in the case of thirty poppies artificially deprived of their petals, as compared with seventy intact poppies, the average visits were as 4.5 is to $2{ }^{\circ}$, the most striking case instanced being that of the Dipterous insect Melanostoma mellina, the visits of which were as 4 is to o.

The experiment and its result does not, to my mind, in the least tend to bear out the theory it is advanced to support, though your correspondent gives the method his approval.

I do not wish to doubt the possibility of smell playing a part in attracting insects, but I certainly cannot see that the artificial removal of the coloured petals proves that colour has no influence. We are fond of attributing great intelligence and power of perception to the bee, and yet in this case the insect is not even given credit for being able to re$\operatorname{cognise}$ what are known to it, from possibly long experience, as the essential parts of the flower! Because we buy well advertised goods, and still continue to buy them when their proved virtue renders advertisement a thing of the past, is it proof that the advertisement played no part in determining our choice? The answer is obvious.

The greater number of insects visiting the poppies shorn of their petals might easily be accounted for, especially in the case of the Diptera, by the presence of some attractive substance in the sap exuded from the cut tissues, and probably by the resulting greater accessibility.

As a contrast to this experiment I would mention that of Lord Avebury, which loses none of its significance through being described in a popular magazine (the I.ondon, Christmas number). Quantities of honey were taken and laid on glass slips, and a marked bee was trained to come to a certain spot for it. The honey was supplied on slips of six different colours-blue, red, yellow, orange, green and white -and on one plain slip. Lord Avebury so arranged matters that the bee was persuaded to visit each and every slip before returning to the hive, the method being as follows:-

Seven slips in a row on lawn; the bee arrives and alights on (say) blue; it is allowed to remain for a few seconds and then driven off, the blue slip being withdrawn; it then goes to (say) white; after a few seconds at white the bee is again driven off, and goes to (say) yellow, the white slip being also withdrawn; after having visited all the slips in this way, and being at last deprived of every one, the bee goes back to the hive.

During the bee's absence the glasses are replaced, but in different order, and on the insect's return it is again noted which slip receives first attention.

Out of a hundred such complete rounds Lord Avebury 